Elmer F. Fabito, Jr., MD

Mary Jane Tipayno-Lubos, MD

Felixberto D. Ayahao, MD

Department of Ear Nose Throat - Head and Neck Surgery Baguio General Hospital and Medical Center
Correspondence: Dr. Felixberto D. Ayahao Department of Otorhinolaryngology Head and Neck Surgery

Baguio General Hospital and Medical Center

Governor Pack Road, Baguio City, Benguet 2600

Philippines

Telefax: (074) 4444176

Email: entbaguio@yahoo.com

The authors declared that this represents original material that is not being considered for publication or has not been published or accepted for publication elsewhere, in full or in part, in print or electronic media; that the manuscript has been read and approved by the authors, that the requirements for authorship have been met by each author, and that each author believes that the manuscript represents honest work.

Disclosures: The authors signed disclosures that there are no financial or other (including personal) relationships, intellectual passion, political or religious beliefs, and institutional affiliations that might lead to a conflict of interest.

Presented at the Philippine Society of Otolaryngology-Head and Neck Surgery, Interesting Case Contest. June 2, 2015. Menarini Office, 4/F W Building, BGC Taguig City.

\title{
Tuberculosis of the Thyroid
}

\begin{abstract}
Objective: To present a case of thyroid tuberculosis and to discuss its clinical presentation, differential diagnoses and management.
\end{abstract}

\section{Methods:}

$\begin{array}{ll}\text { Design: } & \text { Case Report } \\ \text { Setting: } & \text { Tertiary Government Hospital } \\ \text { Patient: } & \text { One }\end{array}$

Results: A 55-year-old farmer presented with an 8-month progressively enlarging anterior neck mass, and fine needle aspiration biopsy yielded grossly turbid straw-colored aspirate admixed with blood with microscopy showing scattered inflammatory cells and macrophages set against a colloid background. After total thyroidectomy, hispathology revealed parenchymal infiltration by multiple aggregates of plump spindled to epitheloid cells forming granulomas with interspersed multinucleated giant cells, central caseation necrosis and surrounding fibrosis with chronic inflammatory infiltrates. The nodal masses also showed prominent germinal centers with interspersed epitheloid cells and foamy macrophages. Final diagnosis was chronic granulomatous inflammation consistent with tuberculosis.

Conclusion: Tuberculosis (TB) of the thyroid is a rare occurrence that can present as inflammation, infection or tumor formation of the thyroid gland. Diagnosis depends on identification of the tubercle from tissues and aspirates by acid fast staining and TB culture. Treatment consists of multiple drug therapy for tuberculosis but thyroidectomy may be an option if the thyroid gland is severely diseased.

Keywords: tuberculosis, endocrine; thyroid disease

Tuberculosis (TB) is one of the leading causes of morbidity and mortality affecting one-third of the world's population. ' It has been reported to occur in many parts of the human body but thyroid gland involvement is extremely rare and its true incidence is unknown. 'Thyroid tuberculosis is rare even in countries in which tuberculosis constitutes an endemic disorder, barely 200 cases have been reported in the world literature. 2 Tuberculosis of the thyroid can present as inflammation, infection or tumor formation of the thyroid gland. Bacteriological studies are needed to make a specific diagnosis. ${ }^{2}$ 


\section{CASE REPORT}

A 55-year-old male farmer from Pangasinan, Philippines was admitted on February 12, 2014 with a chief complaint of anterior neck mass. He was apparently in good general condition until 8 months prior to admission when he noticed a non-tender, soft, approximately golf ball sized mass in the anterior neck over the left lobe of the thyroid with no associated dysphagia, dyspnea, palpitations or tremors. Despite two unrecalled medications and regular follow-up with a private physician, the mass persistently enlarged.

He consulted in our institution 2 months prior to admission. Thyroid hormone test results were normal but neck ultrasonography revealed bilaterally enlarged thyroid lobes with cystic mass lesions measuring 30 $\times 23 \times 11 \mathrm{~mm}$ and $40 \times 38 \times 32 \mathrm{~mm}$ in the right and left lobe, respectively. The cysts exhibited internal comet tail signs reflective of benign colloid lesions. Fine needle aspiration biopsy on the left anterolateral neck mass yielded grossly turbid straw-colored aspirate admixed with blood. Microscopy showed scattered inflammatory cells and macrophages set against a colloid background. Cell block revealed few inflammatory cells scattered in fibrinous background consistent with benign cystic fluid.

The patient was admitted for total thyroidectomy with a clinical impression of multinodular non-toxic goiter. Intraoperative findings revealed a multinodular thyroid gland with approximate combined size of $5 \times 5 \times 2 \mathrm{~cm}$ with firm yellow nodules in both lobes and isthmus with the largest nodule on the left lobe measuring approximately $3 \times 2 \times 3$ $\mathrm{cm}$. The rest of the cut specimen showed a meaty surface. The postoperative course was unremarkable.

Hispathology revealed the left thyroid lobe parenchyma to be infiltrated by multiple aggregates of plump spindled to epitheloid cells forming granulomas with interspersed multinucleated giant cells, central caseation necrosis and surrounding fibrosis with chronic inflammatory infiltrates. The nodal masses attached to the isthmus and right thyroid lobe also showed prominent germinal centers with interspersed epitheloid cells and foamy macrophages. Microsection of the right thyroid lobe revealed varisized, well circumscribed nodules of small to cystically dilated follicles containing scant to abundant colloid and lined by flattened to cuboidal lining cells. The isthmus appeared histologically unremarkable. The final histopathologic diagnosis was chronic granulomatous inflammation consistent with tuberculosis, left thyroid lobe and lymph nodes attached to the right thyroid lobe and isthmus; nodular hyperplasia, right thyroid lobe; no histopathologic change, isthmus. He was started on a course of anti-TB drugs.

\section{DISCUSSION}

Tuberculosis of the thyroid gland whether primary or secondary, is an extremely rare disease with only isolated reports, and a small number of case series have been reported in the literature even in countries endemic for TB. ${ }^{3-4}$ This may be attributed to the resistance of the thyroid gland to infections due to a number of factors, namely, a prosperous lymphatic and vascular supply, well developed capsule, high iodine content of the gland and bactericidal effect of the colloid and iodine.-9 lodine can interact on the outermost layer of microbial cells producing significant effect on their viability. In most cases, although dysphagia, dyspnea and more rarely dysphonia are the main symptoms of the disease, the patient may be asymptomatic as our patient was. ${ }^{10}$ In thyroid tuberculosis, the duration of presenting symptoms varies from 2 weeks to more than a year and there is no relationship with age or

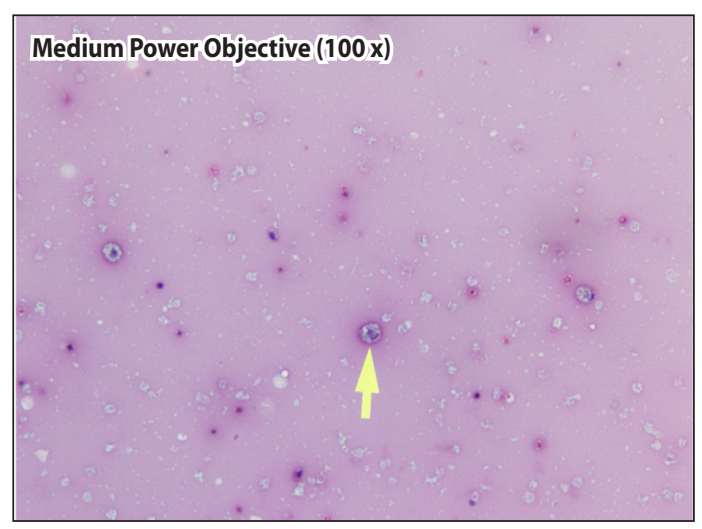

Figure 1. Fine Needle Aspiration cytologic smear, polychromatic stain, medium power objective $(100 \mathrm{x})$, arrow pointing at a macrophage.
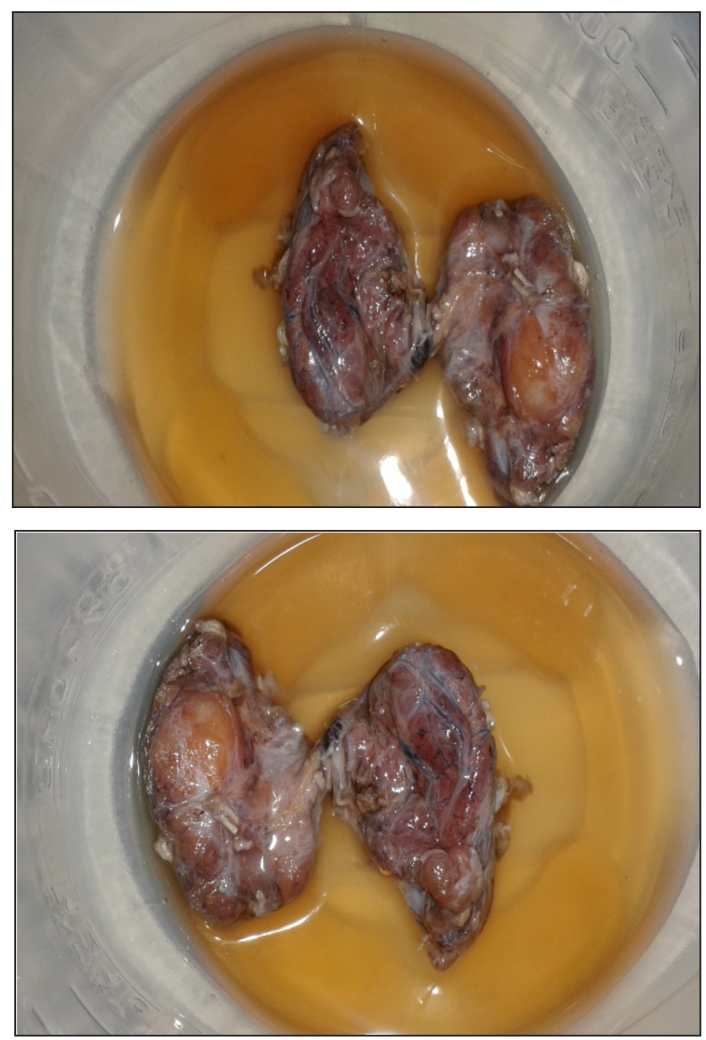

Figure 2. Gross specimen of the thyroid gland, showing multiple complex nodules in both thyroid lobes 


\section{CASE REPORTS}

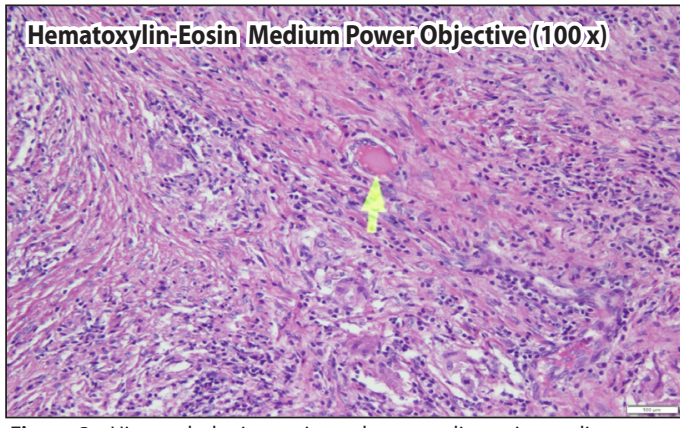

Figure 3. Histopathologic specimen, hematoxylin-eosin, medium power objective (100 x), arrow pointing at multinucleated giant cell

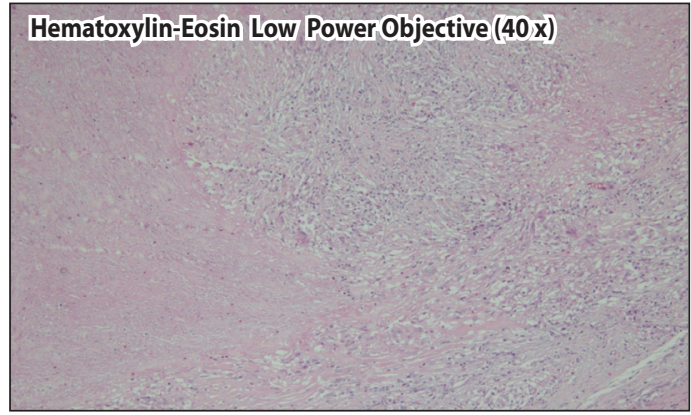

Figure 4. Histopathologic slide, hematoxylin and eosin, low power objective (40x), showing caseation necrosis.

sex. ${ }^{11}$ The most frequent clinical presentation is a solitary thyroid nodule that may present with a cystic component. ${ }^{12}$ Sometimes the patients present with thyrotoxicosis, hypothyroidism, thyroid abscess, thyroid enlargement mimicking cancer, or show signs of subacute granulomatous thyroiditis (De Quervain's) or of chronic non-suppurative thyroiditis. $^{13-16}$ In the majority of cases, patients are euthyroid just like in our case., ${ }^{9,10}$ Imaging techniques are not helpful in establishing the diagnosis. Ultrasonography mostly shows a heterogeneous, hypoechogenic mass that may include cystic degeneration ${ }^{9,17}$ which is similar to our case. Contrast-enhanced computed tomography (CT) may reveal a necrotic centre with a peripheral rim enhancement related to the caseous lesion along with regional lymphadenopathy. ${ }^{17}$ A CT scan was not done in our case as it is not routinely requested for thyroid masses unless mediastinal extension is entertained. Tuberculous thyroiditis can mimic many pathologies. ${ }^{2}$ Localized pain is a predominant symptom and facilitates differential diagnosis in such cases. ${ }^{2}$ Other pathologies to consider are infectious thyroiditis and subacute granulomatous thyroiditis (De Quervain's and thyroid sarcoidosis). ${ }^{18}$ In the event that pain is absent, tuberculous thyroiditis can be mistaken for carcinoma ${ }^{19}$ with which it may coexist. 5,11,20

Tuberculosis of the thyroid gland may be primary or occur in association with tuberculous infection of other organs. ' In such cases, the thyroid is affected by the spread of bacilli via hematogenous or lymphatic routes or directly from the larynx or cervical lymphadenitis. ${ }^{2}$ Tuberculosis primarily affecting the thyroid gland is much more rare and predictably more difficult to diagnose.

The diagnosis is mainly by fine-needle aspiration cytology, a diagnostic step that helps to avoid unnecessary surgery. ${ }^{2}$ Tuberculous lymphadenitis may manifest as a neck mass. Fine needle aspiration (FNA) specimens may yield cytologic evidence consistent with tuberculosis including granulomatous inflammation and/or caseation necrosis. Multiple recent studies have demonstrated greater than $90 \%$ accuracy in diagnosis of tuberculous lymphadenitis with FNA. ${ }^{19,21}$ An improvement in technique to yield more positive acid-fast bacilli (AFB) and culture results from aspirate is called Forin which uses a large bore needle for aspiration of abscess, digesting it with sodium hydroxide and centrifuging it to concentrate microbes at the base of the tube. ${ }^{19}$ In most cases definitive diagnosis is made post-operatively by means of histopathological examination of the surgical specimen as in our patient. ${ }^{7,22}$ Since granulomatous lesions are not pathognomonic of tuberculosis (as they may be seen in sarcoidosis and subacute thyroiditis), caseating necrosis, if present, confirms the diagnosis of tuberculosis. ${ }^{10,22,23}$ As in our case, the left thyroid lobe parenchyma was infiltrated by multiple aggregates of plump spindled to epitheloid cells forming granulomas, with interspersed multinucleated giant cells, central caseation necrosis and surrounding fibrosis with chronic inflammatory infiltrates. The nodal masses attached to the isthmus and right thyroid lobe also showed prominent germinal centers with interspersed epitheloid cells and foamy macrophages. The demonstration of AFB in the gland by ZiehI Nelsen stain can also validate the diagnosis. ${ }^{2}$ However, the mycobacteria are rarely recognised by the stain. ${ }^{2}$ Furthermore, the surgical specimen is rarely submitted for culture unless an infectious process is suspected. ${ }^{2}$ Other investigative modalities include chest $x$-ray, sputum analysis, polymerase chain reaction and cultures with labelled compounds. ${ }^{1}$

Treatment includes anti-TB drugs combined with surgical removal of the affected parts of the thyroid gland or surgical drainage with a good outcome. ${ }^{2}$ For early minor cases, drugs alone are sufficient. In our patient, the affected lobe was also resected and post-operative multiple TB drug therapy was instituted in accordance with our national TB control program. ${ }^{2}$

Thyroid tuberculosis is difficult to diagnose or to differentiate from other thyroid masses due to non-specific signs and symptoms as mentioned in this case. If a patient presents with a strong history of exposure to tuberculosis with cervical lymph adenopathy and fever with a concomitant anterior neck mass that is cystic, tender, erythematous and with abscess formation, then thyroid TB should be considered. ${ }^{2}$ Unfortunately, these were not present in our case.

The routine diagnostic tests performed on our patient did not help us in making the diagnosis either. If there is a high index of suspicion based on physical examination and strong history of exposure, it is 


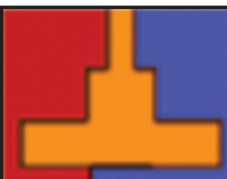

\section{CASE REPORTS}

recommended that Erythrocyte Sedimentation Rate (ESR) and chest $x$-ray should be done. ${ }^{1}$ The most helpful diagnostic test is an ultrasound guided FNA and microbiological tests. ${ }^{2}$

Because we had not made the diagnosis of TB preoperatively and with multiple nodules in both lobes, our patient underwent total thyroidectomy. He was subsequently prescribed anti-TB drugs following the histopathologic report. Appropriate treatment still consists of antiTB drugs since it is now recognized that complete resolution usually follows an appropriate antituberculous drug treatment only. The total duration of chemotherapy was 8 months with favourable outcome. ${ }^{2}$ Surgical intervention is recommended in cases with large abscess or a grossly diseased thyroid gland. ${ }^{2}$

\section{REFERENCES}

1. Mpikashe P, Sathekge MM, Mokgoro NP. Tuberculosis of the thyroid gland: a case report. South Afr Fam Pract. 2004; 46:19-20.

2. Zendah I, Daghfous H, Ben Mrad S, Tritar F. Primary tuberculosis of the thyroid gland. Hormones (Athens). 2008 Oct-Dec; 7(4): 330-3. PMID: 19121995.

3. Collar FA, Huggins CB, 1926 Tuberculosis of the thyroid gland. Ann Surg 84: 804-820

4. Kukreja HK, Sharma ML. Primary tuberculosis of the thyroid gland. Ind J Surg. 1982; 44:190.

5. Al-Mulhim AA, Zakaria HM, Abdel Hadi MS, Al-Mulhim FA, Al-Tamimi DM, Wosornu L. Thyroid tuberculosis mimicking carcinoma: report of two cases. Surg Today. 2002;32(12): 1064-1067. DOI: 10.1007/s005950200214; PMID: 12541023.

6. Dawka S, Jayakumar J, Ghosh A. Primary tuberculosis of the thyroid gland. Kathmandu Univ Med J. 2007 Jul-Sep; 5(3):405-7. PMID: 18604064

7. Bulbuloglu E, Ciralik H, Okur E, Ozdemir G, Ezberci F, Cetinkaya A. Tuberculosis of the thyroid gland: review of the literature. World J Surg. 2006 Feb; 30(2):149-55. DOI: 10.1007/s00268-005 0139-1; PMID: 16425087

8. Mpikashe P, Sathekge MM, Mokgoro NP. Tuberculosis of the thyroid gland: a case report. South Afr Fam Pract. 2004; 46:19-20.

9. Terzidis K, Tourli P, Kiapekou E, Alevizaki M. Thyroid Tuberculosis. Hormones (Athens). 2007 JanMar; 6(1): 75-9. PMID: 17324921

10.Abdulsalam F, Abdulaziz S, Mallik AA. Primary tuberculosis of the thyroid gland. Kuwait Med J. 2005; 37: 116-118.

11. Alan R, O'Flynn W, Clarke SE. Tuberculosis of the thyroid bed presenting as recurrent medullary thyroid carcinoma. Tubercle. 1990 Dec;71(4): 301-302. PMID: 2267683.

12.Khan EM, Haque I, Pandey R, Mishra SK, Sharma AK. Tuberculosis of the thyroid gland: a clinicopathological profile of four cases and review of the literature. Aust N Z J Surg. 1993 Oct;63(10): 807-810. PMID: 8274125

13.Das DK, Pant CS, Chachra KL, Gupta AK. Fine needle aspiration cytology diagnosis of tuberculous thyroiditis. A report of 8 cases. Acta Cytol. 1992 Jul-Aug:36(4): 517-522. PMID: 1636345.

14.Johnson AG, Phillips ME, Thomas RJ. Acute tuberculous abscess of the thyroid gland. Br J Surg. 1973 Aug; 60(8): 668-9. PMID: 4724211

15.Ghosh A, Saha S, Bhattacharya B, Chattopadhay S. Primary tuberculosis of thyroid gland: a rare case report. Am J Otolaryngol. 2007 Jul-Aug; 28(4): 267-270. DOI: 10.1016/j.amjoto.2006.09.005; PMID: 17606045

16.Barnes P, Weatherstone R. Tuberculosis of the thyroid. Two case reports. Br J Dis Chest. 1979 Apr; 73(2):187-191. PMID: 119548

17. Kang BC, Lee SW, Shim SS, Choi HY, Baek SY, Cheon YJ. US and CT findings of tuberculosis of the thyroid gland: three case reports. Clin Imaging. 2000 Sep-Oct;24(5): 283-286. PMID: 11331157.

18. Talwar VK, Gupta H, Kumar A. Isolated tuberculous thyroiditis. JIACM. 2003;4(3): 238-239.

19. Maharjan M, Hirachan S, Kafle PK, Bista M, Shrestha S, Toran KC, et al. Incidence of tuberculosis in enlarged neck nodes, our experience. Kathmandu Univ Med J (KUMJ). 2009 Jan-Mar; 7(25):54-8 PMID: 19483454.

20.Magboo ML, Clark OH. Primary tuberculous thyroid abscess mimicking carcinoma diagnosed by fine needle aspiration biopsy. West J Med. 1990 Dec;153(6): 657-659. PMID: 2127330; PMCID: PMC1002655.

21.Khan R, Harris SH, Verma AK, Syed A. Cervical lymphadenopathy: scrofula revisited. J Laryngol Otol. 2009 Jul;123(7):764-7. DOI: 10.1017/S0022215108003745; PMID: 18845038.

22.El Malki HO, Mohsine R, Benkhraba K, Amahzoune M, Benkabbou A, El Absi M, et al. Thyroid tuberculosis. Diagnosis and treatment. Chemotherapy 2006;52(1): 46-49. DOI: 10.1159/000090244; PMID: 16340200.

23. Kabiri H, Atoini F, Zidane A. Thyroid tuberculosis. Ann Endocrinol (Paris). 2007 Jun;68(2-3): 196198. DOI: 10.1016/j.ando.2007.04.007; PMID: 17532284 